\title{
MESOPOROUS SILICA THIN FILMS FOR BIOMOLECULES SERS DETECTION
}

\author{
Anastasia PERMINOVA, Oleksiy LYUTAKOV, Vaclav ŠVORČIK \\ University of Chemistry and Technology Prague, Czech Republic, EU, perminoa@vscht.cz
}

https://doi.org/10.37904/nanocon.2019.8619

\begin{abstract}
The functional plasmon active surfaces was designed and constructed for advanced Raman spectroscopy purposes. There is a huge demand in biochemistry and medicine for on-line observation of microorganism's behavior, including the biofilms formation. For this goal the Raman spectroscopy measurements can be used. The obstacle we face is that the (bio)liquid may contain a lot of different sized biomolecules. To cut-off unnecessary molecules we propose the utilization of molecular sieve - mesoporous silica thin film (MSTF) deposited on plasmon active surface (periodical gold grating). Our biosensors detect trace amounts of cytotoxic ions and molecules, released from biomaterials. The plasmonic surface was functionalized with MSTF with the aim to provide the selective interaction/entrapping of the targeted objects of interest. Proposed approach also allows to directly monitor cell behavior on the variously modified surfaces and, moreover, surfaces with different morphologies. Development of proposed ideas and concepts introduces an innovative approach in the analysis of complex (bio)molecules system, get new information about the microorganism behavior as well as shed light on previously unknown phenomena in the microbiology field.
\end{abstract}

Keywords: Mesoporous silica, SERS, plasmon-active surface

\section{INTRODUCTION}

Plasmonics represents an unique approach for creation of several analytical components, favourable by extreme sensitivity (the absolute detection limit can be achieved - single molecule recognition), wide usability (plasmon-based sensor as component can be used as a part of spectroscopic system, prepared in the form of fiber probes for local sensing, integrated into the flow system and so on) and reliability [1,2]. Among the various plasmon-based analytical approaches, two main directions can be distinguished: the sensors on the base of the shift of plasmon resonance wavelength position as a function of surrounding refractive index changes, referred to as surface plasmon sensor (SPR), or utilization of plasmon surface-induced enhancement of traditionally weak Raman signal, referred to as surface-enhanced Raman spectroscopy (SERS) approach $[3,4]$. Development of both approaches makes a revolution in the field of analytical chemistry and biochemistry, as well as many other related fields, where the measurement's results provide principally new information or significantly reduce the complexity or required detection route [5]. On the other hand, the growing demands in the field of microbiology, implantology, medicine and other (bio)sciences require the creation of more and more sensitive systems for the analysis of complex samples or recognition of vanishingly small concentrations of targeted molecules in complex samples. Plasmonics is able to give a satisfactory answer to many of these requirements, through the further development and optimization of the plasmon structures. For example, the functionalization of plasmon surfaces with mesoporous silica can cut off large biomolecules and let small ones reach surface and be detected. Mesoporous silica represents a new class of materials containing periodic arrays of channels and cavities, that are tunable over the range $20-100 \AA$ [6].

\section{RESULTS AND DISCUSSION}

\subsection{Surface characterisation}

The main idea of our work is follow: application of SERS provided by Au grating can detect small amounts of targeted molecules, but (bio)samples analysing face a problem - very interfered and difficult to interpret 
spectra. All molecules - big and small - absorb on plasmon-active substrate surface and contribute to SERS signal. The solution we find attractive is mesoporous silica thin film (MSTF). When covering substrates with MSTF before measuring, this addition layer is supposed to work as sieve. Since MSTF got porous structure it can cut-off molecules bigger than pore width and let smaller molecule flow through MSTF and reach grating surface. So that only small (targeted) molecules will give SERS signal. Additionally, we can sort (bio)molecules near SERS surface by adjusting pore size. To claim it we change different MSTF deposition conditions (deposition time, voltage, silica precursor to detergent ratio etc).

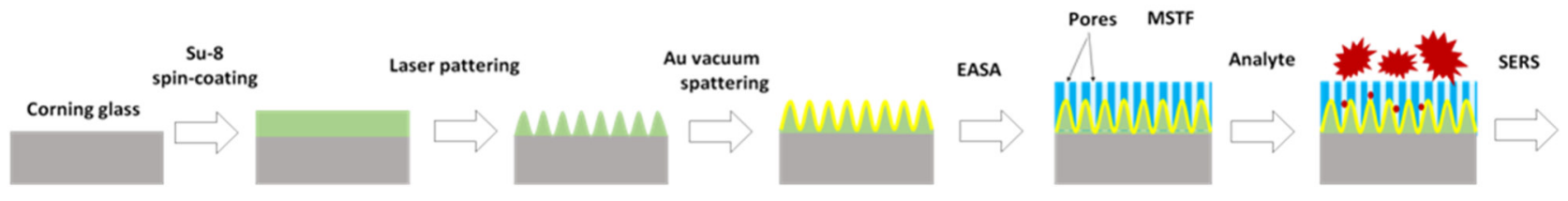

Figure 1 Schematic representation of mesoporous silica substrate preparation
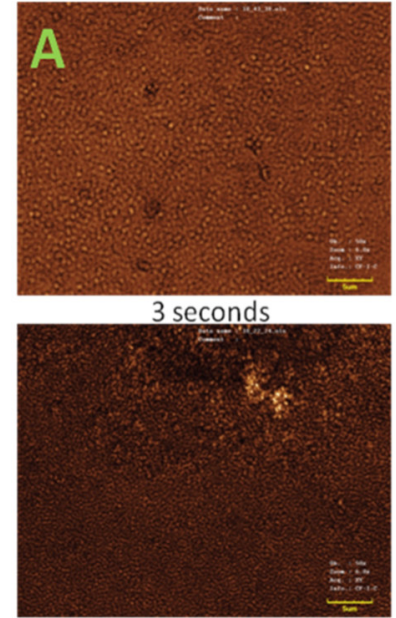

10 seconds

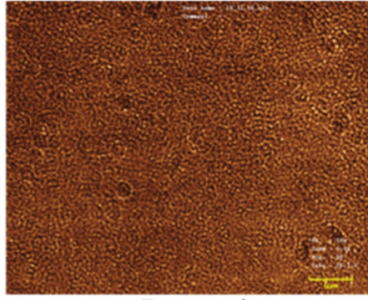

7 seconds

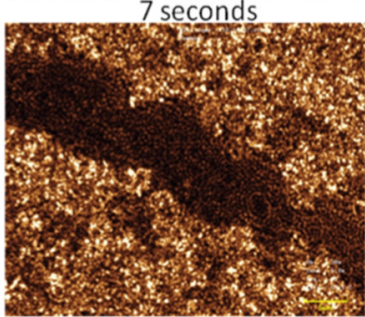

30 seconds
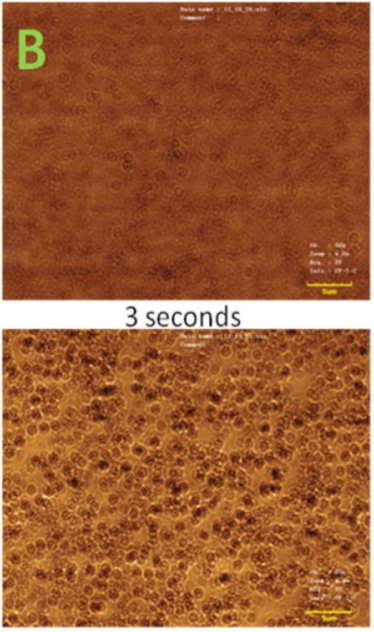

10 seconds
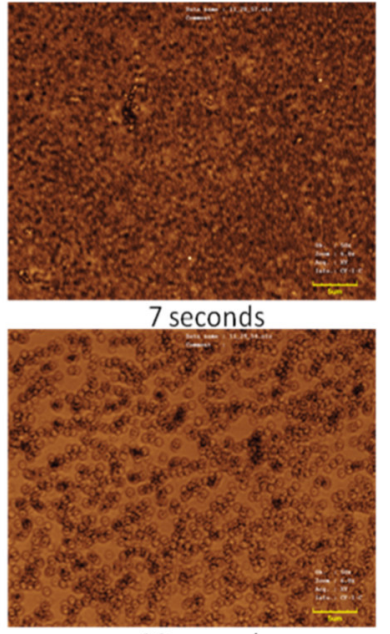

30 seconds

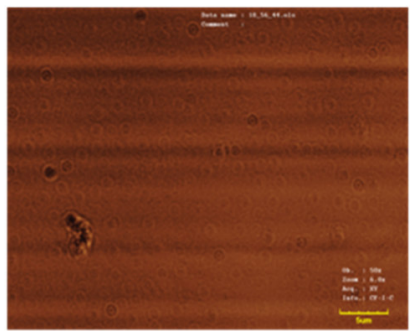

Pristine

Figure 2 Confocal microscopy images of substrates with mesoporous silica deposited for different time.

Samples were made from solution with CTAB:TEOS ratio $0.32(\mathrm{~A})$ and $0.5(\mathrm{~B})$.

To characterise silica surface structure the confocal microscopy was applied. Results are presented in Figure 2 and very smooth surface is evident before MSTF deposition. After 3 seconds deposition both types ( 0.5 and 0.32 Si-coating solutions) the continual homogeneous silica film is formed. For 0.32 ration and 7 seconds deposition the MSTF film keeps being even, but for 0.5 ration we already can observe the formation of aggregates (dark dense spots). The formation of aggregates becomes more noticeable with deposition time increase. 10 and 30 seconds of depositions results in surfaces that are very heterogeneous with many aggregates merging together. Formation of aggregates and high roughness is undesirable outcome since aggregates can block pores so that analyte molecules will not reach gold grating surface. For those reasons, we restricted the deposition time with up to 7 second. 
There is another reason why we do not need to deposit our substrates for too long time. We performed scratchtests using the AFM technique to determine thickness of silica films and how fast it grows. After results evaluation we determined that an average speed of silica growth is $7,5 \mathrm{~nm} / \mathrm{s}$. Film thickness should be around $20 \mathrm{~nm}$ so molecules will not stuck in pores and easily reach plasmonic surface.

So, based on the confocal and AFM microscopies results we came to a conclusion that deposition time for mesoporous silica thin films on Au sputtered substrate should be no more than 3 seconds. Consequently with 3 seconds deposition or less we will get homogeneous, continual film with no aggregates on top of it and with optimal film thickness.

More detailed investigation of surface morphology was performed by SEM. Results are presented in Figure 3. From those images we see aggregates on top of silica surface which are subsequently washed away with ethanol/HCl solution. Moreover SEM results prove that silica stays on surface after interaction with ethanol and CTAB removing.
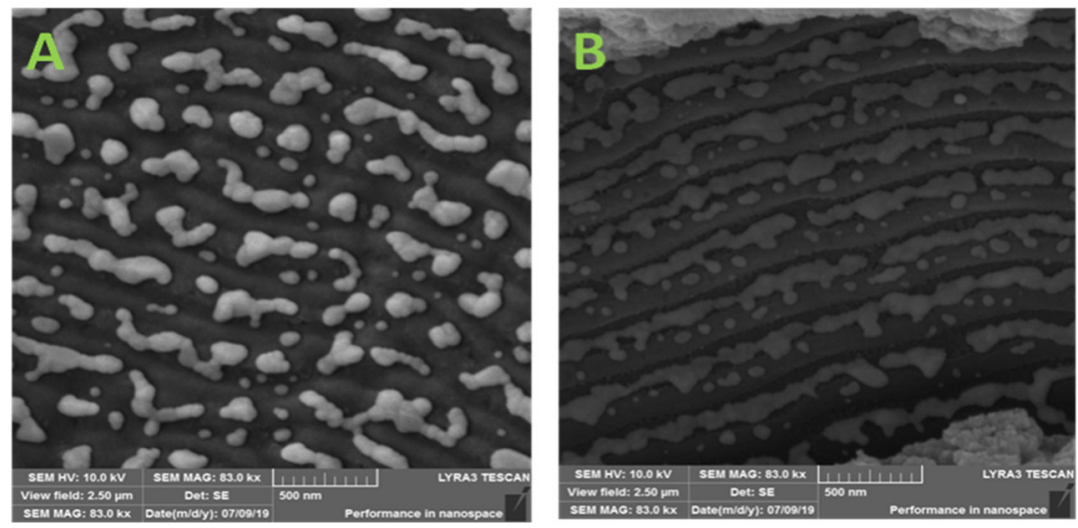

Figure 3 SEM images of MSTF from 0.32 solution before (A) and after (B) surfactant removal

\subsection{Surfactant removal}

To prove CTAB removal with acidic ethanol solution we did SEM-EDX measurements (Table 1). In particular, the Table 1 show the EDX-measured surface elements concentration before and after CTAB removing. Their relative comparison allows us to state CTAB was successfully removed, since there are no nitrogen and bromine on samples surfaces after washing in ethanol/HCl solution. Also we see reduction of carbon which can also occur due to surfactant removal.

Table 1 SEM-EDX results for MSTF substrates before and after CTAB template removal

\begin{tabular}{|c|c|c|c|c|c|c|}
\hline \multirow{2}{*}{ Element } & \multicolumn{3}{|c|}{ Before removal } & \multicolumn{3}{c|}{ After removal } \\
\cline { 2 - 7 } & $\begin{array}{c}\text { Apparent } \\
\text { Concentration }\end{array}$ & $\mathbf{W t} \%$ & $\begin{array}{c}\mathbf{W t} \% \\
\text { Sigma }\end{array}$ & $\begin{array}{c}\text { Apparent } \\
\text { Concentration }\end{array}$ & $\mathbf{W t \%}$ & $\begin{array}{c}\mathbf{W t} \% \\
\text { Sigma }\end{array}$ \\
\hline $\mathrm{C}$ & 1.08 & 51.28 & 1.57 & 0.42 & 33.62 & 0.59 \\
\hline $\mathrm{N}$ & 0.24 & 3.24 & 1.24 & 0 & 0 & 0 \\
\hline $\mathrm{O}$ & 1.52 & 20.18 & 0.83 & 2.53 & 37.57 & 0.45 \\
\hline $\mathrm{Al}$ & 0.03 & 0.26 & 0.9 & 0.01 & 0.08 & 0.1 \\
\hline $\mathrm{Si}$ & 1.63 & 15.78 & 0.56 & 2.21 & 28.73 & 0.33 \\
\hline $\mathrm{Br}$ & 0.67 & 8.68 & 1.8 & 0 & 0 & 0 \\
\hline $\mathrm{Mo}$ & 0.04 & 0.57 & 0.76 & 0 & 0 & 0 \\
\hline $\mathrm{T}$ Total & & 100 & & & 100 & \\
\hline
\end{tabular}


Removal of CTAB was also confirmed by IR spectroscopy. IR spectra were taken before and after samples immersion in ethanol solution. As can be expected the spectrum of pristine sample (Au grating, before MSTF deposition) is plane, without any characteristic features. In turn, CTAB IR spectrum, measured in powder and presented for comparison has two pronounced characteristic peaks, located at 2920 and $2850 \mathrm{~cm}^{-1}$. These characteristic peaks are clearly seen on Au grating/MSTF spectrum before CTAB removing. After the immersion in ethanol/HCl solution the CTAGB characteristic peaks disappear, but very wide and broad peak appears confirming presence of alcohol groups remaining after extraction of surfactant. Disappearance of these band (and conservation of $\mathrm{SiO}_{2}$ structure - see SEM-EDX result) indicate successful removing CTAB while keeping silica on Au grating surface.

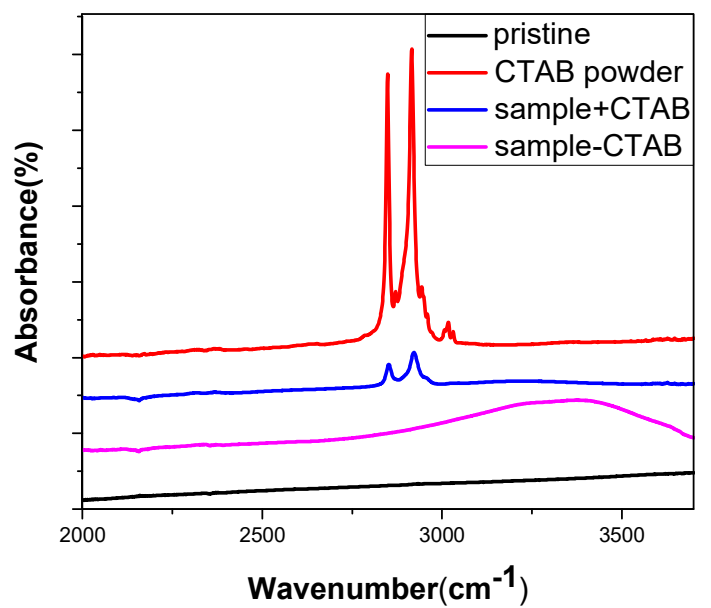

Figure 4 FTIR spectra of pristine substrate, pure CTAB, and samples before and after CTAB removal

\subsection{SERS with analyte standard}

After finding the optimal MSTF deposition conditions the SERS measurements were performed in the next step. At the first stage the model SERS analyte - crystal violet (CV) was used. The CV was dissolved in water to obtain $10^{-6} \mathrm{~mol} / \mathrm{l}$ concentration and drop-deposited on Au/MSTF surface. Results of SERS measurements are presented in Figure 5. Appearance of characteristic SERS signal from both $\mathrm{CV}$ and $\mathrm{SiO}_{2}$ indicate analyte's ability to reach the Au grating surface and that silica is still remaining after CTAB removal.

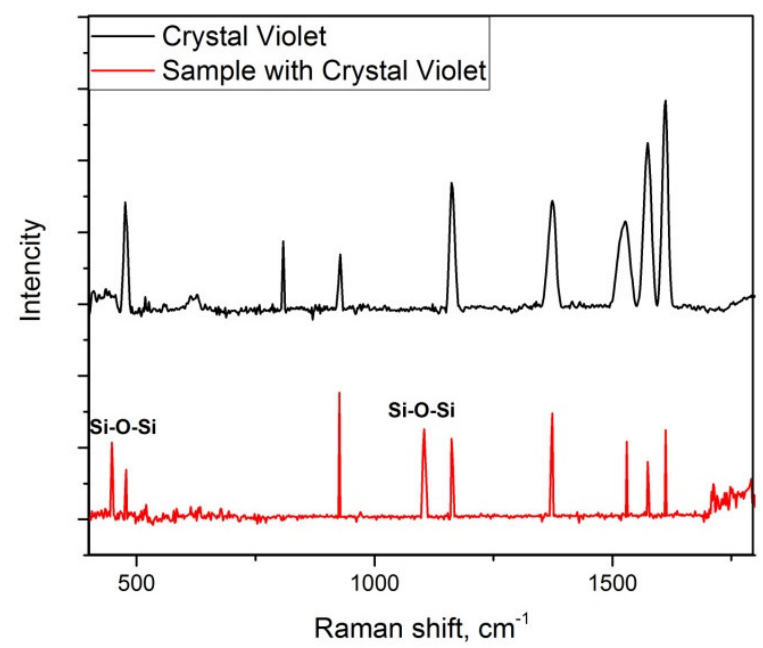

Figure 5 Raman spectra of crystal violet powder and MSTF sample with drop-deposited crystal violet on it 
So, using the described parameters we created MS structure, able to allow to small molecules reach the plasmonic active surface. Our future work will be focused on proving MS ability to cut-off big molecules while letting small ones be detected in the context of peptide solution with analyte dye. Further we will be analysing more complex bio samples containing different sized molecules e.g. bacterial strains and films

\section{CONCLUSION}

To prevent interfered and difficult to interpret spectra when analysing complex bio samples mesoporous silica thin films were synthesised on Au-grating substrate. The conditions of MSTF deposition were carefully optimized. The created functional SERS substrates were involved in SERS measurements of model analyte. Our future work will be focused on proving MS ability to cut-off big molecules while letting small ones be detected in the context of peptide solution with analyte dye. Further we will be analysing more complex bio samples containing different sized molecules e.g. bacterial strains and films

\section{ACKNOWLEDGEMENTS}

\section{This work was financially supported by the internal Grant Agency UCT № 1260881911}

\section{REFERENCES}

[1] SHARMA, A.K., PANDEY, A.K., KAUR, B. Simulation study on comprehensive sensing enhancement of BlueP/MoS 2 - and BlueP/WS 2 -based fluoride fiber surface plasmon resonance sensors: analysis founded on damping, field, and optical power, Applied Optics. 2019, 58, pp. 4518-4525.

[2] LIU, B., MONSHAT, H., GU, Z., LU, M., ZHAO, X. Recent advances in merging photonic crystals and plasmonics for bioanalytical applications. Analyst. 2018, 143, pp. 2448-2458.

[3] SINGH, P. SPR Biosensors: Historical Perspectives and Current Challenges. Sensors and Actuators B: Chemical. 2016, 229, pp. 110-130.

[4] POWELL, J.A., VENKATAKRISHNAN, K., TAN B. Hybridized enhancement of the SERS detection of chemical and bio-marker molecules through Au nanosphere ornamentation of hybrid amorphous/crystalline Si nanoweb nanostructure biochip devices. Journal of Materials Chemistry B. 2016, 4, pp. 5713-5728.

[5] GRANGER, J.H., SCHLOTTER, N.E., CRAWFORD, A.C., PORTER, M.D. Prospects for point-of-care pathogen diagnostics using surface-enhanced Raman scattering (SERS). Chemical Society Reviews. 2016, 45, pp. 38653882.

[6] KRESGE, C.T., LEONOWICZ, M.E., ROTH, J.J., VARTULI, J.C., BECK, J.S. Ordered Mesoporous Molecular Sieves Synthesized by Liquid-Crystal Template Mechanism. Nature. 1992, 359, pp. 710-712.

[7] KALACHYOVA, Y., MARES D., LYUTAKOV, O., KOSTEIN M., LAPCAK, L., SVORCIK, V. Surface plasmon polaritons on silver gratings for optimal SERS response. J. Phys. Chem. C, 2015, 119, pp. 9506-9512

[8] GUSELNIKOVA O., POSTNIKOV, P., TRELIN, A., SVORCIK, V., LYUTAKOV, O. Dual Mode Chip Enantioselective Express Discrimination of Chiral Amines via Wettability-Based Mobile Application and Portable Surface-Enhanced Raman Spectroscopy Measurements. ACS Sensors. 2019, 4, pp. 1032-1039.

[9] WALCARIUS, A., SIBOTTIER E., ETIENNE, M., GHANBAJA, J. Electrochemically assisted self-assembly of mesoporous silica thin films. Nature Materials. 2007, 6, pp. 602-608. 